\title{
Convergence properties of generalized Lupaş-Kantorovich operators
}

\author{
Qasim M. ${ }^{1, \bigotimes}$, Khan A. ${ }^{2}$, Abbas Z. ${ }^{1}$, Mursaleen M. ${ }^{2,3}$
}

In the present paper, we consider the Kantorovich modification of generalized Lupaş operators, whose construction depends on a continuously differentiable, increasing and unbounded function $\rho$. For these new operators we give weighted approximation, Voronovskaya type theorem, quantitative estimates for the local approximation.

Key words and phrases: Lupas operator, Kantorovich operator, Korovkin type theorem, convergence theorem, Voronovskaya type theorem.

\footnotetext{
${ }^{1}$ Baba Ghulam Shah Badshah University, Rajouri-185234, Jammu and Kashmir, India

2 Aligarh Muslim University, Aligarh 202002, India

${ }^{3}$ China Medical University, Taichung, Taiwan

$\square$ Corresponding author

E-mail: bgsbuqasimegmail. com(Qasim M.), asifjnu07@gmail.com(Khan A.), zam@bgsbu.ac. in (Abbas Z.), mursaleenm@gmail.com (Mursaleen M.)
}

\section{Introduction}

Classical approximation theory, including polynomial approximation is a fundamental research area in applied mathematics. Development in approximation theory play an important role in numerical solution of partial differential equation, image processing as well as data science and many other disciplines. For example, radial basis functions and shift invariant spaces are widely used for geometric modeling in aerospace and automobile industries.

In 1912, S.N. Bernstein [7] proposed the famous polynomials, constructed by probabilistic method, to give the simple, short and most elegant proof of Weierstrass theorem [25], namely

$$
\mathscr{B}_{m}(g ; u)=\sum_{j=0}^{m} b_{m, j}(u) g\left(\frac{j}{m}\right)
$$

where $B_{m}: C[0,1] \rightarrow C[0,1], u \in[0,1], m=1,2, \ldots$, and the Bernstein basis $b_{m, j}$ is defined by

$$
b_{m, j}(u)=\left(\begin{array}{c}
m \\
j
\end{array}\right) u^{j}(1-u)^{m-j}
$$

Bernstein polynomials are not useful for discontinuous measureable functions, more suitable modification of the Bernstein polynomials, for this kind of functions, that was introduced by L.V. Kantorovich [17], namely, for any $g \in L_{P}[0,1], u \in[0,1]$ and $p \geq 1$

$$
\mathscr{K}_{m}(g ; u)=(m+1) \sum_{j=0}^{m} b_{m, j}(u) \int_{j /(m+1)}^{(j+1) /(m+1)} g(y) d y,
$$

$\mathrm{y} \Delta \mathrm{K} 517.518 .8$

2020 Mathematics Subject Classification: 41A10, 41A25, 41A36. 
where $b_{m, j}(u)$ is given by $(1)$.

After that Kantorovich type modification of several sequences of linear positive operators has been made and studied for their approximation behavior (cf. [11, 23, 24]). Several researchers also defined different types of generalizations of these operators and studied their approximation properties, we refer the reader to e.g. [1,6, 8, 16, 20-22] etc.

In 2011, D. Cárdenas-Morales, P. Garrancho and I. Raşa [9] introduced Bernstein-type operators defined for $f \in C[0,1]$ by $B_{m}\left(g o \rho^{-1}\right) \rho \rho, B_{m}$ being the classical Bernstein operators and $\rho$ being any function that is continuously differentiable $\infty$ times on $[0,1]$, such that $\rho(0)=0, \rho(1)=1$ and $\rho^{-1}(u)>0$ for $u \in[0,1]$. The results obtained there showed that approximation with these new construction of Bernstein operators are sensitive and present better convergence results with the suitable selection of $\rho$. A Durrmeyer type generalization of $B_{m}\left(g o \rho^{-1}\right) o \rho$ was also studied in [4]. The results of the aforementioned papers show that it is possible to obtain some improvements of the classical approximation by Bernstein and Bernstein-Durrmeyer operators in certain senses, simultaneously. Inspired by this idea, many researchers have performed studied in this direction. Recently A. Aral, D. Inoan and I. Raşa [5] introduced similar modifications of the Szász-Mirakyan operators further properties in [2], also Szász-Durrmeyer operators in [3].

Very recently, a new modification of Lupaş operators [19] has been introduced in [14] by using a suitable function $\rho$, which satisfies following properties:

$\left(\rho_{1}\right) \rho$ is a continuously differentiable function on $[0, \infty)$;

$\left(\rho_{2}\right) \rho(0)=0$ and $\inf _{u \in[0, \infty)} \rho^{\prime}(u) \geq 1$.

The new operators which are called generalized Lupaş operators are defined as

$$
\mathscr{L}_{m}^{\rho}(g ; u)=2^{-m \rho(u)} \sum_{j=0}^{\infty} \frac{(m \rho(u))_{j}}{2^{j} j !}\left(g o \rho^{-1}\right)\left(\frac{j}{m}\right)
$$

for $m \geq 1, u \geq 0$, and suitable function $g$ defined on $[0, \infty)$. If $\rho(u)=u$, then (2) reduces to the Lupaş operators defined in [19].

In this paper, we define Kantorovich variant of operators (2) which depends on $\rho$. In Kantorovich type modifications we mainly replace the sample values $j / m$ by the mean values of $\left(g \circ \rho^{-1}\right)$ in the interval $[j /(m+1),(j+1) /(m+1)]$.

The present work is organized as follows. In the second section, we define Kantorovich variant of the generalized Lupaş operators and calculate their moments and central moments. In the third section, we study convergence properties of new constructed operators in the light of weighted space. In section fourth, we obtain the order of approximation of generalized Lupaş-Kantorovich operators associated with the weighted modulus of continuity. In section fifth, a Voronovskaya type result is obtained. Finally, in last section, we obtain some local approximation results related to $\mathscr{K}$-functional, also we define a Lipschitz-type functions, as well as related results.

\section{Construction of the generalized Lupaş-Kantorovich operators}

Inspired by the above mentioned work, we introduce Kantorovich variant of operators (2), which depend on a suitable function $\rho$ as follows. 
Definition 1. For $g \in L_{1}[0, \infty)$ and $m \in \mathbb{N}$, we define Kantorovich variant of generalized Lupaş operators as

$$
\mathscr{K}_{m}^{\rho}(g ; u)=(m+1) 2^{-m \rho(u)} \sum_{j=0}^{\infty} \frac{(m \rho(u))_{j}}{2^{j} j !} \int_{j /(m+1)}^{(j+1) /(m+1)}\left(g \circ \rho^{-1}\right)(y) d y,
$$

where the rising factorial $(m \rho(u))_{j}$ is defined as:

$$
(m \rho(u))_{0}=1, \quad(m \rho(u))_{j}=(m \rho(u))(m \rho(u)+1)(m \rho(u)+2) \ldots(m \rho(u)+j-1), \quad j \geq 0 .
$$

The operators (3) are linear and positive. The operators $\mathscr{K}_{m}^{\rho}$ are constructed to obtain results in approximation for discontinuous functions on the basis of integral mean of $\left(g \circ \rho^{-1}\right)$ over small intervals. Next, we prove some auxiliary results for $\mathscr{K}_{m}^{\rho}$, which are used to prove main results.

Lemma 1. Let $\mathscr{K}_{m}^{\rho}$ be given by (3). Then for each $u \geq 0$ and $m \in \mathbb{N}$ we have:

(i)

$$
\mathscr{K}_{m}^{\rho}(1 ; u)=1
$$

(ii)

$$
\mathscr{K}_{m}^{\rho}(\rho ; u)=\frac{m \rho(u)}{m+1}+\frac{1}{2(m+1)}
$$

(iii)

$$
\mathscr{K}_{m}^{\rho}\left(\rho^{2} ; u\right)=\frac{m^{2} \rho^{2}(u)}{(m+1)^{2}}+\frac{3 m \rho(u)}{(m+1)^{2}}+\frac{1}{3(m+1)^{2}}
$$

(iv)

$$
\mathscr{K}_{m}^{\rho}\left(\rho^{3} ; u\right)=\frac{m^{3} \rho^{3}(u)}{(m+1)^{3}}+\frac{15 m^{2} \rho^{2}(u)}{2(m+1)^{3}}+\frac{10 m \rho(u)}{(m+1)^{3}}+\frac{1}{4(m+1)^{3}} ;
$$

(v)

$$
\mathscr{K}_{m}^{\rho}\left(\rho^{4} ; u\right)=\frac{m^{4} \rho^{4}(u)}{(m+1)^{4}}+\frac{14 m^{3} \rho^{3}(u)}{(m+1)^{4}}+\frac{50 m^{2} \rho^{2}(u)}{(m+1)^{4}}+\frac{43 m \rho(u)}{(m+1)^{4}}+\frac{1}{5(m+1)^{4}}
$$

Proof. (i)

$$
\mathscr{K}_{m}^{\rho}(1 ; u)=\frac{m+1}{2^{m \rho(u)}} \sum_{j=0}^{\infty} \frac{(m \rho(u))_{j}}{2^{j} j !} \int_{j /(m+1)}^{(j+1) /(m+1)} d y=\frac{m+1}{2^{m \rho(u)}} 2^{m \rho(u)} \frac{1}{m+1}=1 .
$$

(ii)

$$
\begin{aligned}
\mathscr{K}_{m}^{\rho}(\rho ; u) & =\frac{m+1}{2^{m \rho(u)}} \sum_{j=0}^{\infty} \frac{(m \rho(u))_{j}}{2^{j} j !} \int_{j /(m+1)}^{(j+1) /(m+1)} y d y \\
& =\frac{m+1}{2^{m \rho(u)}} \sum_{j=0}^{\infty} \frac{(m \rho(u))_{j}}{2^{j} j !} \frac{j}{(m+1)^{2}} \\
& =\frac{2^{-m \rho(u)}}{(m+1)} \sum_{j=0}^{\infty} \frac{(m \rho(u))_{j}}{2^{j} j !} j=\frac{m \rho(u)}{(m+1)}+\frac{1}{2(m+1)} .
\end{aligned}
$$


(iii)

$$
\begin{aligned}
\mathscr{K}_{m}^{\rho}\left(\rho^{2} ; u\right)= & \frac{m+1}{2^{m \rho(u)}} \sum_{j=0}^{\infty} \frac{(m \rho(u))_{j}}{2^{j} j !} \int_{j /(m+1)}^{(j+1) /(m+1)} y^{2} d y \\
= & \frac{m+1}{2^{m \rho(u)}} \sum_{j=0}^{\infty} \frac{(m \rho(u))_{j}}{2^{j} j !} \frac{3 j^{2}+3 j+1}{3(m+1)^{3}} \\
= & \frac{2^{-m \rho(u)}}{3(m+1)^{2}} \sum_{j=0}^{\infty} \frac{(m \rho(u))_{j}}{2^{j} j !}\left(3 j^{2}+3 j+1\right)+\frac{2^{-m \rho(u)}}{3(m+1)^{2}} \sum_{j=0}^{\infty} \frac{(m \rho(u))_{j}}{2^{j} j !} 3 j^{2} \\
& +\frac{2^{-m \rho(u)}}{3(m+1)^{2}} \sum_{j=0}^{\infty} \frac{(m \rho(u))_{j}}{2^{j} j !} 3 j+\frac{2^{-m \rho(u)}}{3(m+1)^{2}} \sum_{j=0}^{\infty} \frac{(m \rho(u))_{j}}{2^{j} j !} \\
= & \frac{m^{2} \rho^{2}(u)}{(m+1)^{2}}+\frac{3 m \rho(u)}{(m+1)^{2}}+\frac{1}{3(m+1)^{2}} .
\end{aligned}
$$

(iv)

$$
\begin{aligned}
\mathscr{K}_{m}^{\rho}\left(\rho^{3} ; u\right)= & \frac{m+1}{2^{m \rho(u)}} \sum_{j=0}^{\infty} \frac{(m \rho(u))_{j}}{2^{j} j !} \int_{j /(m+1)}^{(j+1) /(m+1)} y^{3} d y \\
= & \frac{m+1}{2^{m \rho(u)}} \sum_{j=0}^{\infty} \frac{(m \rho(u))_{j}}{2^{j} j !} \frac{4 j^{3}+6 j^{2}+4 j+1}{4(m+1)^{4}} \\
= & \frac{2^{-m \rho(u)}}{4(m+1)^{3}} \sum_{j=0}^{\infty} \frac{(m \rho(u))_{j}}{2^{j} j !}\left(4 j^{3}+6 j^{2}+4 j+1\right)+\frac{2^{-m \rho(u)}}{3(m+1)} \sum_{j=0}^{\infty} \frac{(m \rho(u))_{j}}{2^{j} j !} 4 j^{3} \\
& +\frac{2^{-m \rho(u)}}{3(m+1)} \sum_{j=0}^{\infty} \frac{(m \rho(u))_{j}}{2^{j} j !} 6 j^{2}+\frac{2^{-m \rho(u)}}{3(m+1)} \sum_{j=0}^{\infty} \frac{(m \rho(u))_{j}}{2^{j} j !} 4 j+\frac{2^{-m \rho(u)}}{3(m+1)} \sum_{j=0}^{\infty} \frac{(m \rho(u))_{j}}{2^{j} j !} \\
= & \frac{m^{3} \rho^{3}(u)}{(m+1)^{3}}+\frac{15 m^{2} \rho^{2}(u)}{2(m+1)^{3}}+\frac{10 m \rho(u)}{(m+1)^{3}}+\frac{1}{4(m+1)^{3}} .
\end{aligned}
$$

(v)

$$
\begin{aligned}
\mathscr{K}_{m}^{\rho}\left(\rho^{4} ; u\right) & =\frac{m+1}{2^{m \rho(u)}} \sum_{j=0}^{\infty} \frac{(m \rho(u))_{j}}{2^{j} j !} \int_{j /(m+1)}^{(j+1) /(m+1)} y^{4} d y \\
& =\frac{m+1}{2^{m \rho(u)}} \sum_{j=0}^{\infty} \frac{(m \rho(u))_{j}}{2^{j} j !} \frac{5 j^{4}+10 j^{3}+10 j^{5} j+1}{5(m+1)^{5}} \\
& =\frac{2^{-m \rho(u)}}{5(m+1)^{4}} \sum_{j=0}^{\infty} \frac{(m \rho(u))_{j}}{2^{j} j !}\left(5 j^{4}+10 j^{3}+10 j^{5} j+1\right) \\
& =\frac{m^{4} \rho^{4}(u)}{(m+1)^{4}}+\frac{14 m^{3} \rho^{3}(u)}{(m+1)^{4}}+\frac{50 m^{2} \rho^{2}(u)}{(m+1)^{4}}+\frac{43 m \rho(u)}{(m+1)^{4}}+\frac{1}{5(m+1)^{4}} .
\end{aligned}
$$

Corollary 1. By using Lemma 1 and by linearity of operators $\mathscr{K}_{m}^{\rho}$, we can acquire the central moments as:

(i)

$$
\mathscr{K}_{m}^{\rho}(\rho(\zeta)-\rho(u) ; u)=\frac{1-2 \rho(u)}{2(m+1)} ;
$$


(ii)

$$
\mathscr{K}_{m}^{\rho}\left((\rho(\zeta)-\rho(u))^{2} ; u\right)=\frac{1}{(m+1)^{2}} \rho^{2}(u)+\frac{2 m-1}{(m+1)^{2}} \rho(u)+\frac{1}{3(m+1)^{2}}
$$

(iii)

$$
\begin{aligned}
\mathscr{K}_{m}^{\rho}((\rho(\zeta) & \left.-\rho(u))^{3} ; u\right)=\frac{m^{3}-3 m(m+1)+3(m+1)^{2}-(m+1)^{3}}{(m+1)^{3}} \rho^{3}(u) \\
& +\frac{15 m^{2}-9 m(m+1)+3(m+1)^{2}}{(m+1)^{2}} \rho^{2}(u)+\frac{9 m-1}{(m+1)^{3}} \rho(u)+\frac{1}{4(m+1)^{3}}
\end{aligned}
$$

(iv)

$$
\begin{gathered}
\mathscr{K}_{m}^{\rho}\left((\rho(\zeta)-\rho(u))^{4} ; u\right)=\frac{m^{4}-4 m^{3}(m+1)+6 m^{2}(m+1)^{2}-4(m+1)^{3}+(m+1)^{4}}{(m+1)^{4}} \rho^{4}(u) \\
+\frac{14 m^{3}+30 m^{2}(m+1)+18 m(m+1)^{2}-2(m+1)^{3}}{(m+1)^{4}} \rho^{3}(u) \\
+\frac{50 m^{2}-40 m(m+1)-2(m+1)^{2}}{(m+1)^{4}} \rho^{2}(u)+\frac{42 m-1}{(m+1)^{4}} \rho(u)+\frac{1}{5(m+1)^{4}} .
\end{gathered}
$$

\section{Weighted approximation}

In this section we prove convergence properties of new constructed operators $\mathscr{K}_{m}^{\rho}$ in the light of weighted space.

Let $\Phi(u)$ be a function satisfying the conditions $\left(\rho_{1}\right)$ and $\left(\rho_{2}\right)$ given above. Also, we take the weight function $\Phi(u)=1+\rho^{2}(u)$ and we define the weighted spaces as follows

$$
\mathscr{B}_{\Phi}[0, \infty)=\left\{g:[0, \infty) \rightarrow \mathbb{R}|| g(u) \mid \leq \mathscr{M}_{g} \Phi(u), u \geq 0\right\},
$$

where $\mathscr{M}_{g}$ is a constant, which depends only on $g . \mathscr{B}_{\Phi}[0, \infty)$ is a normed linear space equipped with the norm

$$
\|g\|_{\Phi}=\sup _{u \in[0, \infty)} \frac{|g(u)|}{\Phi(u)} .
$$

Also, the subspaces $\mathscr{C}_{\Phi}[0, \infty), U_{\Phi}[0, \infty)$ and $U_{\Phi}[0, \infty)$ of $\mathscr{B}_{\Phi}[0, \infty)$ are defined as

$$
\begin{gathered}
\mathscr{C}_{\Phi}[0, \infty)=\left\{g \in \mathscr{B}_{\Phi}[0, \infty): g \text { is continuous on }[0, \infty)\right\}, \\
\mathscr{C}_{\Phi}^{*}[0, \infty)=\left\{g \in \mathscr{C}_{\Phi}[0, \infty): \lim _{u \rightarrow \infty} \frac{g(u)}{\Phi(u)}=\mathscr{M}_{g}=\text { constant }\right\}, \\
U_{\Phi}[0, \infty)=\left\{g \in \mathscr{C}_{\Phi}[0, \infty): \frac{g(u)}{\Phi(u)} \text { is uniformly continuous on }[0, \infty)\right\} .
\end{gathered}
$$

It is obvious that $\mathscr{C}_{\Phi}^{*}[0, \infty) \subset U_{\Phi}[0, \infty) \subset \mathscr{C}_{\Phi}[0, \infty) \subset \mathscr{B}_{\Phi}[0, \infty)$.

In [12], A.D. Gadjiev and A. Aral prove the following results for the weighted Korovkin type theorems. We consider $\left(\mathscr{G}_{m}\right)_{m \geq 1}$ a sequence of positive linear operators, which act from $\mathscr{C}_{\Phi}[0, \infty)$ to $\mathscr{B}_{\Phi}[0, \infty)$.

Lemma 2 ([12]). The positive linear operators $\mathscr{G}_{m}, m \geq 1$, act from $\mathscr{C}_{\Phi}[0, \infty)$ to $\mathscr{B}_{\Phi}[0, \infty)$ if and only if the inequality

$$
\left|\mathscr{G}_{m}(\Phi ; u)\right| \leq \mathscr{M}_{m} \Phi(u), \quad u \geq 0,
$$

holds, where $\mathscr{M}_{m}>0$ is a constant depending on $m$. 
Theorem 1 ([12]). Let the sequence of positive linear operators $\mathscr{G}_{m}, m \geq 1$ act from $\mathscr{C}_{\Phi}[0, \infty)$ to $\mathscr{B}_{\Phi}[0, \infty)$ and satisfy

$$
\lim _{m \rightarrow \infty}\left\|\mathscr{G}_{m} \rho^{i}-\rho^{i}\right\|_{\Phi}=0, \quad i=0,1,2 .
$$

Then for any function $g \in C_{\Phi}^{*}[0, \infty)$ we have

$$
\lim _{m \rightarrow \infty}\left\|\mathscr{G}_{m}(g)-g\right\|_{\Phi}=0 .
$$

Therefore, we can prove the following results.

Theorem 2. For each function $g \in C_{\Phi}^{*}[0, \infty)$ we have

$$
\lim _{m \rightarrow \infty}\left\|\mathscr{K}_{m}^{\rho}(g)-g\right\|_{\Phi}=0 .
$$

Proof. By Lemma 1 (i) and (ii), it is clear that

$$
\left\|\mathscr{K}_{m}^{\rho}(1 ; u)-1\right\|_{\Phi}=0
$$

and

$$
\left\|\mathscr{K}_{m}^{\rho}(\rho ; u)-\rho\right\|_{\Phi}=\left|\frac{m}{m+1}-1\right| \sup _{u \in[0, \infty)} \frac{\rho(u)}{1+\rho^{2}(u)}+\frac{1}{2(m+1)} \leq \frac{3}{2(m+1)} .
$$

Again by Lemma 1 (iii), we have

$$
\begin{aligned}
\left\|\mathscr{K}_{m}^{\rho}\left(\rho^{2} ; u\right)-\rho^{2}\right\|_{\Phi}= & \left|\frac{m^{2}}{(m+1)^{2}}-1\right| \sup _{u \in[0, \infty)} \frac{\rho^{2}(u)}{1+\rho^{2}(u)} \\
& +\frac{3 m}{(m+1)^{2}} \sup _{u \in[0, \infty)} \frac{\rho(u)}{1+\rho^{2}(u)}+\frac{1}{3(m+1)^{2}} \leq \frac{15 m+4}{3(m+1)^{2}} .
\end{aligned}
$$

Then, from Lemma 1 and (4) we get

$$
\lim _{m \rightarrow \infty}\left\|\mathscr{K}_{m}^{\rho}\left(\rho^{i}\right)-\rho^{i}\right\|_{\Phi}=0, \quad i=0,1,2 .
$$

Hence, the proof is completed.

\section{Rate of convergence}

In this section, we determine the rate of convergence for $\mathscr{K}_{m}^{\rho}$ by weighted modulus of continuity $\omega_{\rho}(g ; \delta)$, which was recently considered by A. Holhoş [15] as follows

$$
\omega_{\rho}(g ; \delta)=\sup _{u, \zeta \in[0, \infty),|\rho(\zeta)-\rho(u)| \leq \delta} \frac{|g(\zeta)-g(u)|}{\Phi(\zeta)+\Phi(u)}, \quad \delta>0,
$$

where $g \in \mathscr{C}_{\Phi}[0, \infty)$, with the following properties:

(i) $\omega_{\rho}(g ; 0)=0$;

(ii) $\omega_{\rho}(g ; \delta) \geq 0, \delta \geq 0$ for $g \in \mathscr{C}_{\Phi}[0, \infty)$;

(iii) $\lim _{\delta \rightarrow 0} \omega_{\rho}(g ; \delta)=0$ for each $g \in U_{\Phi}[0, \infty)$. 
Theorem 3 ([15]). Let $\mathscr{G}_{m}: \mathscr{C}_{\Phi}[0, \infty) \rightarrow \mathscr{B}_{\Phi}[0, \infty)$ be a sequence of positive linear operators with

$$
\begin{aligned}
& \left\|\mathscr{G}_{m}\left(\rho^{0}\right)-\rho^{0}\right\|_{\Phi^{0}}=a_{m}, \quad\left\|\mathscr{G}_{m}(\rho)-\rho\right\|_{\Phi^{1 / 2}}=b_{m}, \\
& \left\|\mathscr{G}_{m}\left(\rho^{2}\right)-\rho^{2}\right\|_{\Phi}=c_{m}, \quad\left\|\mathscr{G}_{m}\left(\rho^{3}\right)-\rho^{3}\right\|_{\Phi^{3 / 2}}=d_{m},
\end{aligned}
$$

where the sequences $a_{m}, b_{m}, c_{m}$ and $d_{m}$ converge to zero as $m \rightarrow \infty$. Then

$$
\left\|\mathscr{G}_{m}(g)-g\right\|_{\Phi^{3 / 2}} \leq\left(7+4 a_{m}+2 c_{m}\right) \omega_{\rho}\left(g ; \delta_{m}\right)+\|g\|_{\Phi} a_{m},
$$

for all $g \in \mathscr{C}_{\Phi}[0, \infty)$, where

$$
\delta_{m}=2 \sqrt{\left(a_{m}+2 b_{m}+c_{m}\right)\left(1+a_{m}\right)}+a_{m}+3 b_{m}+3 c_{m}+d_{m} .
$$

Theorem 4. Let for each $g \in \mathscr{C}_{\Phi}[0, \infty)$ we have

$$
\left\|\mathscr{K}_{m}^{\rho}(g)-g\right\|_{\Phi^{3 / 2}} \leq \frac{21 m^{2}+72 m+39}{3(m+1)^{2}} \omega_{\rho}\left(g ; \delta_{m}\right),
$$

where

$$
\delta_{m}=2 \sqrt{\frac{24 m+13}{3(m+1)^{2}}}+\frac{120 m^{2}+142 m+27}{4(m+1)^{3}} .
$$

Proof. If we calculate the sequences $\left(a_{m}\right),\left(b_{m}\right),\left(c_{m}\right)$ and $\left(d_{m}\right)$, then by using Lemma 1 , clearly we have

$$
\begin{gathered}
\left\|\mathscr{K}_{m}^{\rho}\left(\rho^{0}\right)-\rho^{0}\right\|_{\Phi^{0}}=0=a_{m} \\
\left\|\mathscr{K}_{m}^{\rho}(\rho)-\rho\right\|_{\Phi^{1 / 2}} \leq \frac{3}{2(m+1)}=b_{m}
\end{gathered}
$$

and

$$
\left\|\mathscr{K}_{m}^{\rho}\left(\rho^{2}\right)-\rho^{2}\right\|_{\Phi} \leq \frac{15 m+4}{3(m+1)^{2}}=c_{m}
$$

Finally,

$$
\left\|\mathscr{K}_{m}^{\rho}\left(\rho^{3}\right)-\rho^{3}\right\|_{\Phi^{3 / 2}} \leq \frac{42 m^{2}+4 m+5}{4(m+1)^{3}}=d_{m} .
$$

Thus, the conditions (5) are satisfied. Now by Theorem 3, we obtain the desired result.

Remark 1. From property (iii) of $\omega_{\rho}(g ; \delta)$ and Theorem 4, we have

$$
\lim _{m \rightarrow \infty}\left\|\mathscr{K}_{m}^{\rho}(g)-g\right\|_{\Phi^{3 / 2}}=0 \quad \text { for } \quad g \in U_{\Phi}[0, \infty) .
$$

\section{Voronovskaya type theorem}

In this section, we prove pointwise convergence of $\mathscr{K}_{m}^{\rho}$ by using a technique, which is developed in [9] by D. Cárdenas-Morales, P. Garrancho and I. Raşa.

Theorem 5. Let $g \in \mathscr{C}_{\Phi}[0, \infty), u \in[0, \infty)$ and suppose that $\left(g o \rho^{-1}\right)^{\prime}$ and $\left(g o \rho^{-1}\right)^{\prime \prime}$ exist at $\rho(u)$. If $\left(g \circ \rho^{-1}\right)^{\prime \prime}$ is bounded on $[0, \infty)$, then we have

$$
\lim _{m \rightarrow \infty} m\left[\mathscr{K}_{m}^{\rho}(g ; u)-g(u)\right]=\rho(u)\left(g o \rho^{-1}\right)^{\prime}\left\{\frac{1-2 \rho(u)}{2}\right\}+\rho(u)\left(g o \rho^{-1}\right)^{\prime \prime} \rho(u) .
$$


Proof. By using Taylor expansion of $\left(g \circ \rho^{-1}\right)$ at $\rho(u) \in[0, \infty)$ we have

$$
\begin{aligned}
g(\zeta)=\left(g o \rho^{-1}\right)(\rho(\zeta))= & \left(g o \rho^{-1}\right)(\rho(u))+\left(g o \rho^{-1}\right)^{\prime}(\rho(u))(\rho(\zeta)-\rho(u)) \\
& +\frac{\left(g o \rho^{-1}\right)^{\prime \prime}(\rho(u))(\rho(\zeta)-\rho(u))^{2}}{2}+\lambda_{u}(\zeta)(\rho(\zeta)-\rho(u))^{2},
\end{aligned}
$$

where

$$
\lambda_{u}(\zeta)=\frac{\left(g o \rho^{-1}\right)^{\prime \prime}(\rho(\zeta))-\left(g o \rho^{-1}\right)^{\prime \prime}(\rho(u))}{2} .
$$

Therefore, by the assumption on $g$ and (7) ensures that $\left|\lambda_{u}(\zeta)\right| \leq \mathscr{K}$ for all $\zeta \in[0, \infty)$ and

$$
\lim _{\zeta \rightarrow u} \lambda_{u}(\zeta)=0
$$

Now by applying the operators (3) to the equality (6), we get

$$
\begin{aligned}
{\left[\mathscr{K}_{m}^{\rho}(g ; u)\right.} & -g(u)]=\left(g o \rho^{-1}\right)^{\prime}(\rho(u)) \mathscr{K}_{m}^{\rho}((\rho(\zeta)-\rho(u)) ; u) \\
& +\frac{\left(g o \rho^{-1}\right)^{\prime \prime}(\rho(u)) \mathscr{K}_{m}^{\rho}\left((\rho(\zeta)-\rho(y))^{2} ; u\right)}{2}+\mathscr{K}_{m}^{\rho}\left(\lambda^{u}(\zeta)\left((\rho(\zeta)-\rho(u))^{2} ; u\right)\right) \zeta .
\end{aligned}
$$

From Lemma 1 and Corollary 1, we obtain

$$
\lim _{m \rightarrow \infty} m \mathscr{K}_{m}^{\rho}((\rho(\zeta)-\rho(u)) ; u)=\frac{1-2 \rho(u)}{2}
$$

and

$$
\lim _{m \rightarrow \infty} m \mathscr{K}_{m}^{\rho}\left((\rho(\zeta)-\rho(u))^{2} ; u\right)=2 \rho(u) .
$$

By estimating the last term on the right hand side of equality (8), we will get the proof.

Since from (7), for every $\epsilon>0$,

$$
\lim _{\zeta \rightarrow u} \lambda_{u}(\zeta)=0
$$

Let $\delta>0$ such that $\left|\lambda_{u}(\zeta)\right|<\epsilon$ for every $\zeta \geq 0$. By Cauchy-Schwartz inequality, we get

$$
\begin{aligned}
\lim _{m \rightarrow \infty} m \mathscr{K}_{m}^{\rho}\left(\left|\lambda_{u}(\zeta)\right|(\rho(\zeta)-\rho(u))^{2} ; u\right) \leq & \epsilon \lim _{m \rightarrow \infty} m \mathscr{K}_{m}^{\rho}\left((\rho(\zeta)-\rho(u))^{2} ; u\right) \\
& +\frac{\mathscr{K}}{\delta^{2}} \lim _{m \rightarrow \infty} \mathscr{K}_{m}^{\rho}\left((\rho(w)-\rho(u))^{4} ; u\right) .
\end{aligned}
$$

Since

$$
\lim _{m \rightarrow \infty} m \mathscr{K}_{m}^{\rho}\left((\rho(\zeta)-\rho(u))^{4} ; u\right)=0
$$

we obtain

$$
\lim _{m \rightarrow \infty} m \mathscr{K}_{m}^{\rho}\left(\left|\lambda_{u}(\zeta)\right|(\rho(\zeta)-\rho(u))^{2} ; u\right)=0
$$

Thus, by taking into account the equations (9), (10) and (11) to equation (8) this prove the theorem. 


\section{Local and global Approximation}

In order to prove local approximation theorems for the operators $\mathscr{K}_{m}^{\rho}$, let us recall some basic concepts and results concerning modulus of continuity and $K$-functional. Let $\mathscr{C}_{B}[0, \infty)$ be the space of real-valued continuous and bounded functions $g$ defined on the interval $[0, \infty)$. The norm $\|\cdot\|$ on the space $\mathscr{C}_{B}[0, \infty)$ is given by

$$
\|g\|=\sup _{0 \leq u<\infty}|g(u)| .
$$

Further, $K$-functional is defined as

$$
K_{2}(g, \delta)=\inf _{r \in W^{2}}\left\{\|g-r\|+\delta\left\|g^{\prime \prime}\right\|\right\}
$$

where $\delta>0$ and $W^{2}=\left\{s \in \mathscr{C}_{B}[0, \infty): r^{\prime}, r^{\prime \prime} \in \mathscr{C}_{B}[0, \infty)\right\}$. Then, in view of known result [10], there exists an absolute constant $\mathscr{D}>0$ such that $\mathscr{K}(g, \delta) \leq \mathscr{D} \omega_{2}(g, \sqrt{\delta})$.

For $g \in C_{B}[0, \infty)$ the second order modulus of smoothness is defined as follows

$$
\omega_{2}(g, \sqrt{\delta})=\sup _{0<h \leq \sqrt{\delta}} \sup _{u \in[0, \infty)}|g(u+2 h)-2 g(u+h)+g(u)|
$$

and the usual modulus of continuity is defined as

$$
\omega(g, \delta)=\sup _{0<h \leq \delta} \sup _{u \in[0, \infty)}|g(u+h)-g(u)| .
$$

Theorem 6. Let $g \in \mathscr{C}_{B}[0, \infty)$ and $\rho$ be a function satisfying the conditions $\left(\rho_{1}\right),\left(\rho_{2}\right)$ and $\left\|\rho^{\prime \prime}\right\|$ is finite. Then, there exists an absolute constant $\mathscr{D}>0$ such that

$$
\left|\mathscr{K}_{m}^{\rho}(g ; u)-g(u)\right| \leq \mathscr{D} K\left(g, \delta_{m}(u)\right)
$$

where

$$
\delta_{m}(u)=\left\{\frac{1}{(m+1)^{2}} \rho^{2}(u)+\frac{2 m-1}{(m+1)^{2}} \rho(u)+\frac{1}{3(m+1)^{2}}\right\} .
$$

Proof. Let $r \in W^{2}$ and $u, y \in[0, \infty)$. By using Taylor's formula we have

$$
r(y)=r(u)+\left(r o \rho^{-1}\right)^{\prime}(\rho(u))(\rho(y)-\rho(u))+\int_{\rho(u)}^{\rho(y)}(\rho(y)-v)\left(r o \rho^{-1}\right)^{\prime \prime}(v) d v .
$$

We use the equality

$$
\left(r o \rho^{-1}\right)^{\prime \prime}(\rho(u))=\frac{r^{\prime \prime}(u)}{\left(\rho^{\prime}(u)\right)^{2}}-r^{\prime \prime}(u) \frac{\rho^{\prime \prime}(u)}{\left(\rho^{\prime}(u)\right)^{3}} .
$$

Now, put $v=\rho(z)$ in the last term in equality (12), we get

$$
\begin{aligned}
\int_{\rho(u)}^{\rho(y)} & (\rho(y)-v)\left(r o \rho^{-1}\right)^{\prime \prime}(v) d v=\int_{u}^{y}(\rho(y)-\rho(z)) \frac{r^{\prime \prime}(z) \rho^{\prime}(z)-r^{\prime}(z) \rho^{\prime \prime}(v)}{\left(\rho^{\prime}(z)\right)^{2}} d z \\
& =\int_{\rho(u)}^{\rho(y)}(\rho(y)-v) \frac{r^{\prime \prime}\left(\rho^{-1}(v)\right)}{\left(\rho^{\prime}\left(\rho^{-1}(v)\right)^{2}\right.} d v-\int_{\rho(u)}^{\rho(y)}(\rho(y)-v) \frac{r^{\prime}\left(\rho^{-1}(v)\right) \rho^{\prime \prime}\left(\rho^{-1}(v)\right)}{\left(\rho^{\prime}\left(\rho^{-1}(v)\right)^{3}\right.} d v .
\end{aligned}
$$


By applying the operator (3) to the both sides of equality (12) and by using Lemma 1 and (13), we deduce

$$
\begin{aligned}
\mathscr{K}_{m}^{\rho}(r ; u)=r(u) & +\mathscr{K}_{m}^{\rho}\left(\int_{\rho(u)}^{\rho(y)}(\rho(y)-v) \frac{r^{\prime \prime}\left(\rho^{-1}(v)\right)}{\left(\rho^{\prime}\left(\rho^{-1}(v)\right)^{2}\right.} d v ; u\right) \\
& -\mathscr{K}_{m}^{\rho}\left(\int_{\rho(u)}^{\rho(y)}(\rho(y)-v) \frac{r^{\prime}\left(\rho^{-1}(v)\right) \rho^{\prime \prime}\left(\rho^{-1}(v)\right)}{\left(\rho^{\prime}\left(\rho^{-1}(v)\right)^{3}\right.} d v ; u\right) .
\end{aligned}
$$

As we know $\rho$ is strictly increasing on $[0, \infty)$ and with condition $\left(\rho_{2}\right)$, we get

$$
\left|\mathscr{K}_{m}^{\rho}(r ; u)-r(u)\right| \leq \mathscr{M}_{m, 2}^{\rho}(u)\left(\left\|r^{\prime \prime}\right\|+\left\|r^{\prime}\right\|\left\|\rho^{\prime \prime}\right\|\right),
$$

where

$$
\mathscr{M}_{m, 2}^{\rho}(u)=\mathscr{K}_{m}^{\rho}\left((\rho(y)-\rho(u))^{2} ; u\right) .
$$

For all $g \in \mathscr{C}_{B}[0, \infty)$, we have

$$
\left|\mathscr{K}_{m}^{\rho}(r ; u)\right| \leq\left\|g o \rho^{-1}\right\| 2^{-m \rho(u)} \sum_{j=0}^{\infty} \frac{(m \rho(u))_{j}}{2^{j} j !} \leq\|g\| \mathscr{K}_{m}^{\rho}(1 ; u)=\|g\| .
$$

Hence, we have

$$
\begin{aligned}
& \left|\mathscr{K}_{m}^{\rho}(g ; u)-g(u)\right| \leq\left|\mathscr{K}_{m}^{\rho}(g-r ; u)\right|+\left|\mathscr{K}_{m}^{\rho}(r ; u)-r(u)\right|+|r(u)-g(u)| \\
& \leq 2\|g-r\|+\left\{\frac{1}{(m+1)^{2}} \rho^{2}(u)+\frac{2 m-1}{(m+1)^{2}} \rho(u)+\frac{1}{3(m+1)^{2}}\right\}\left(\left\|r^{\prime \prime}\right\|+\left\|r^{\prime}\right\|\left\|\rho^{\prime \prime}\right\|\right),
\end{aligned}
$$

if we choose $\mathscr{D}=\max \left\{2,\left\|\rho^{\prime \prime}\right\|\right\}$, then

$$
\left|\mathscr{K}_{m}^{\rho}(g ; u)-g(u)\right| \leq \mathscr{D}\left(2\|g-r\|+\left\{\frac{1}{(m+1)^{2}} \rho^{2}(u)+\frac{2 m-1}{(m+1)^{2}} \rho(u)+\frac{1}{3(m+1)^{2}}\right\}\left\|r^{\prime \prime}\right\|_{W^{2}}\right) .
$$

Taking infimum over all $r \in W^{2}$ we obtain

$$
\left|\mathscr{K}_{m}^{\rho}(g ; u)-g(u)\right| \leq \mathscr{D} K\left(g, \delta_{m}(u)\right) \text {. }
$$

Now, we recall the Lipschitz class given in [13]. Let $\rho$ be a function satisfying the conditions $\left(\rho_{1}\right),\left(\rho_{2}\right), 0<\alpha \leq 1$ and $\operatorname{Lip} \mathscr{H}(\rho(u) ; \alpha), \mathscr{H} \geq 0$, is the set of functions $g$ satisfying the inequality

$$
|g(y)-g(u)| \leq \mathscr{H}|\rho(u)-\rho(u)|^{\alpha}, \quad u, y \geq 0 .
$$

Moreover, for a bounded subset $\mathscr{Y} \subset[0, \infty)$, we say that the function $g \in \mathscr{C}_{B}[0, \infty)$ belongs to $\operatorname{Lip}_{\mathscr{H}}(\rho(u) ; \alpha), 0<\alpha \leq 1$, on $\mathscr{Y}$ if

$$
|g(z)-g(u)| \leq \mathscr{H}_{\alpha, g}|\rho(y)-\rho(u)|^{\alpha}, \quad u \in \mathscr{Y} \quad \text { and } \quad y \geq 0,
$$

where $\mathscr{H}_{\alpha, g}$ is a constant depending on $\alpha$ and $g$.

Theorem 7. Let $\rho$ be a function satisfying the conditions $\left(\rho_{1}\right),\left(\rho_{2}\right)$. Then for any function $g \in \operatorname{Lip}_{\mathscr{H}}(\rho(u) ; \alpha), 0<\alpha \leq 1$, and for every $u \in(0, \infty), m \in \mathbb{N}$, we have

$$
\left|\mathscr{K}_{m}^{\rho}(g ; u)-g(u)\right| \leq \mathscr{H}\left(\delta_{m}(u)\right)^{\alpha / 2} \text {, }
$$

where

$$
\delta_{m}(u)=\left\{\frac{1}{(m+1)^{2}} \rho^{2}(u)+\frac{2 m-1}{(m+1)^{2}} \rho(u)+\frac{1}{3(m+1)^{2}}\right\} .
$$


Proof. Assume that $\alpha=1$. Then, for $g \in \operatorname{Lip}_{\mathscr{H}}(\alpha ; 1)$ and $u \in(0, \infty)$, we have

$$
\left|\mathscr{K}_{m}^{\rho}(g ; u)-g(u)\right| \leq \mathscr{K}_{m}^{\rho}(|g(y)-g(u)| ; u) \leq \mathscr{H}^{\rho} \mathscr{K}_{m}^{\rho}(|\rho(y)-g(u)| ; u) .
$$

By applying Cauchy-Schwartz inequality, we get

$$
\left|\mathscr{K}_{m}^{\rho}(g ; u)-g(u)\right| \leq \mathscr{H}\left[\mathscr{K}_{m}^{\rho}\left((\rho(y)-\rho(u))^{2} ; u\right)\right]^{1 / 2} \leq \mathscr{H} \sqrt{\delta_{m}(u)} .
$$

Let us assume that $\alpha \in(0,1)$. Then, for $g \in \operatorname{Lip}_{\mathscr{H}}(\alpha ; 1)$ and $u \in(0, \infty)$, we have

$$
\left|\mathscr{K}_{m}^{\rho}(g ; u)-g(u)\right| \leq \mathscr{K}_{m}^{\rho}(|g(y)-g(u)| ; u) \leq \mathscr{H}^{\rho} \mathscr{K}_{m}^{\rho}\left(|\rho(y)-g(u)|^{\alpha} ; u\right) .
$$

By taking $p=1 / \alpha$ and $q=1 /(1-\alpha), g \in \operatorname{Lip}_{\mathscr{H}}(\rho(u) ; \alpha)$, and applying Hölder's inequality we have

$$
\left|\mathscr{K}_{m}^{\rho}(g ; u)-g(u)\right| \leq \mathscr{H}\left[\mathscr{K}_{m}^{\rho}(\mid(\rho(y)-\rho(u) \mid ; u)]^{\alpha} .\right.
$$

Finally, by applying Cauchy-Schwartz inequality, we get

$$
\left|\mathscr{K}_{m}^{\rho}(g ; u)-g(u)\right| \leq \mathscr{H}\left(\delta_{m}(u)\right)^{\alpha / 2} .
$$

Theorem 8. Let $\rho$ be a function satisfying the conditions $\left(\rho_{1}\right),\left(\rho_{2}\right)$ and $\mathscr{Y}$ be a bounded subset of $[0, \infty)$. Then for any $g \in \operatorname{Lip}_{\mathscr{H}}(\rho(u) ; \alpha), 0<\alpha \leq 1$, on $\mathscr{Y}$, we have

$$
\left|\mathscr{K}_{m}^{\rho}(g ; u)-g(u)\right| \leq \mathscr{H}_{\alpha, g}\left\{\left(\delta_{m}(u)\right)^{\alpha / 2}+2\left[\rho^{\prime}(u)\right]^{\alpha} d^{\alpha}(u, \mathscr{Y})\right\}, \quad u \in[0, \infty), \quad m \in \mathbb{N},
$$

where

$$
d(u, \mathscr{Y})=\inf \{\|u-z\|: z \in \mathscr{Y}\},
$$

$\mathscr{M}_{\alpha, g}$ is a constant depending on $\alpha$ and $g$, and

$$
\delta_{m}(u)=\left\{\frac{1}{(m+1)^{2}} \rho^{2}(u)+\frac{2 m-1}{(m+1)^{2}} \rho(u)+\frac{1}{3(m+1)^{2}}\right\} .
$$

Proof. Let $\overline{\mathscr{Y}}$ be the closure of $\mathscr{Y}$ in $[0, \infty)$. Then there exists a point $u_{0} \in \overline{\mathscr{Y}}$ such that

$$
d(u, \mathscr{Y})=\left|u-u_{0}\right| \text {. }
$$

Using the monotonicity of $\mathscr{K}_{m}^{\rho}$ and the hypothesis of $g$, we obtain

$$
\begin{aligned}
\left|\mathscr{K}_{m}^{\rho}(g ; u)-g(u)\right| & \leq \mathscr{K}_{m}^{\rho}\left(\left|g(y)-g\left(u_{0}\right)\right| ; u\right)+\mathscr{K}_{m}^{\rho}\left(\left|g(u)-g\left(u_{0}\right)\right| ; u\right) \\
& \leq \mathscr{M}_{\alpha, g}\left\{\mathscr{K}_{m}^{\rho}\left(\left|\rho(y)-\rho\left(u_{0}\right)\right|^{\alpha} ; u\right)+\left|\rho(u)-\rho\left(u_{0}\right)\right|^{\alpha}\right\} \\
& \leq \mathscr{H}_{\alpha, g}\left\{\mathscr{K}_{m}^{\rho}\left(|\rho(y)-\rho(u)|^{\alpha} ; u\right)+2\left|\rho(u)-\rho\left(u_{0}\right)\right|^{\alpha}\right\} .
\end{aligned}
$$

By using Hölder's inequality for $p=2 / \alpha$ and $q=2 /(2-\alpha)$, as well as the fact

$$
\left|\rho(u)-\rho\left(u_{0}\right)\right|=\rho^{\prime}(u)\left|\rho(u)-\rho\left(u_{0}\right)\right|
$$

in the last inequality we get

$$
\left|\mathscr{K}_{m}^{\rho}(g ; u)-g(u)\right| \leq \mathscr{H}_{\alpha, g}\left\{\left[\mathscr{K}_{m}^{\rho}\left((\rho(y)-\rho(u))^{2} ; u\right)\right]^{1 / 2}+2\left[\rho^{\prime}(u)\left|\rho(u)-\rho\left(u_{0}\right)\right|\right]^{\alpha}\right\} .
$$

Hence, by Corollary1 we get the proof. 
Now, we recall the local approximation given in [18] for $g \in \mathscr{C}_{B}[0, \infty)$, given as

$$
\widetilde{\omega}_{\alpha}^{\rho}(g ; u)=\sup _{y \neq u, y \in(0, \infty)} \frac{|g(y)-g(u)|}{|y-u|^{\alpha}}, \quad u \in[0, \infty) \quad \text { and } \quad \alpha \in(0,1] .
$$

Then we get the next result.

Theorem 9. Let $g \in \mathscr{C}_{B}[0, \infty)$ and $\alpha \in(0,1]$. Then, for all $u \in[0, \infty)$, we have

$$
\left|\mathscr{K}_{m}^{\rho}(g ; u)-g(u)\right| \leq \widetilde{\omega}_{\alpha}^{\rho}(g ; u)\left(\delta_{m}(u)\right)^{\alpha / 2},
$$

where

$$
\delta_{m}(u)=\left\{\frac{1}{(m+1)^{2}} \rho^{2}(u)+\frac{2 m-1}{(m+1)^{2}} \rho(u)+\frac{1}{3(m+1)^{2}}\right\} .
$$

Proof. We know that

$$
\left|\mathscr{K}_{m}^{\rho}(g ; u)-g(u)\right| \leq \mathscr{K}_{m}^{\rho}(|g(y)-g(u)| ; u) .
$$

From equation (14), we have

$$
\left|\mathscr{K}_{m}^{\rho}(g ; u)-g(u)\right| \leq \widetilde{\omega}_{\alpha}^{\rho}(g ; u) \mathscr{K}_{m}^{\rho}\left(|\rho(y)-\rho(u)|^{\alpha} ; u\right) .
$$

By applying Hölder's inequality with $p=2 / \alpha$ and $q=2 /(2-\alpha)$, we have

$$
\left|\mathscr{K}_{m}^{\rho}(g ; u)-g(u)\right| \leq \widetilde{\omega}_{\alpha}^{\rho}(g ; u)\left[\mathscr{K}_{m, q}^{\rho}\left((\rho(y)-\rho(u))^{2} ; u\right)\right]^{\alpha / 2} \leq \widetilde{\omega}_{\alpha}^{\rho}(g ; u)\left(\delta_{m}(u)\right)^{\alpha / 2},
$$

which proves the desired result

\section{Acknowledgment}

The first author is grateful to Council of Scientific and Industrial Research (CSIR), India, for providing the Senior Research Fellowship with award no. (09/1172(0001)/2017-EMR-I).

\section{References}

[1] Abel U. Asymptotic approximation with Kantorovich polynomial. Approx. Theory Appl. 1998, 14 (3), $106-116$.

[2] Acar T. Asymptotic formulas for generalized Szász-Mirakyan operators. Appl. Math. Comput. 2015, 263, $233-239$. doi:10.1016/j.amc.2015.04.060

[3] Acar T., Ulusoy G. Approximation by modified Szász-Durrmeyer operators. Period. Math. Hungar. 2016, 72 (1), 64-75. doi:10.1007/s10998-015-0091-2

[4] Acar T., Aral A., Raşa I. Modified Bernstein-Durrmeyer operators. Gen. Math. 2014, 22 (1), $27-41$.

[5] Aral A., Inoan D., Raşa I. On the generalized Szász-Mirakyan operators. Results. Math. 2014, 65 (3-4), 441-452. doi:10.1007/s00025-013-0356-0

[6] Acu A.M., Manav N., Sofonea D.F. Approximation properties of $\lambda$-Kantorovich operators. J. Inequal. Appl. 2018, 202 (2018). doi:10.1186/s13660-018-1795-7

[7] Bernstein S.N. Démonstation du théorème de Weierstrass fondée sur le calcul de probabilités. Commun. Soc. Math. Kharkow 1912, 2 série, 13 (1), 1-2.

[8] Cal J., Valle A.M. A generalization of Bernstein-Kantorovich operators. J. Math. Anal. Appl. 2000, 252 (2), $750-766$. doi:10.1006/jmaa.2000.7119

[9] Cárdenas-Morales D., Garrancho P., Raşa I. Bernstein-type operators which preserve polynomials. Comput. Math. Appl. 2011, 62 (1), 158-163. doi:10.1016/j.camwa.2011.04.063 
[10] DeVore R.A., Lorentz G.G. Constructive Approximation. Grundlehren Math. Wiss., Springer-Verlag, Berlin, 1993.

[11] Duman O., Özarslan M.A., Della Vecchia B. Modified Szász-Mirakjan-Kantorovich operators preserving linear functions. Turkish J. Math. 2009, 33, 151-158.

[12] Gadjiev A.D. The convergence problem for a sequence of positive linear operators on unbounded sets, and theorems analogous to that of P.P. Korovkin. Dokl. Akad. Nauk SSSR 1974, 218 (5), 1001-1004. (in Russian)

[13] Gadjiev A.D., Aral A. The estimates of approximation by using a new type of weighted modulus of continuity. Comput. Math. Appl. 2007, 54 (1), 127-135. doi:10.1016/j.camwa.2007.01.017

[14] Gül H., İlarslan İ., Aral A., Başcanbaz-Tunca G. Generalized Lupaş operators. AIP Conf. Proc. 2018,1926 (1), 020019. doi:10.1063/1.5020468

[15] Holhoş A. Quantitative estimates for positive linear operators in weighted space. General Math. 2008, 16 (4), 99-110.

[16] İçöz G. A Kantorovich variant of a new type Bernstein-Stancu polynomials. Appl. Math. Comput. 2012,218 (17), 8552-8560. doi:10.1016/j.amc.2012.02.017

[17] Kantorovich L.V. On some expansions in polynomials in the form of S.N. Bernstein. Dokl. Akad. Nauk SSSR 1930, 21, 563-568; 22, 595-600. (in Russian)

[18] Lenze B. On Lipschitz-type maximal functions and their smoothness spaces. Proc. Netherl. Acad. Sci. A 1988, 91, 53-63.

[19] Lupaş A. The approximation by means of some linear positive operators. In: Müller M.W., Felten M., Mache D.H. (Eds.) Approximation Theory, 86. Akademie Verlag, Berlin, 1995, 201-227.

[20] Mohiuddine S.A., Özger F. Approximation of functions by Stancu variant of Bernstein-Kantorovich operators based on shape parameter $\alpha$. RACSAM 2020, 114 (70).

[21] Mursaleen M., Ahasan M. The Dunkl generalization of Stancu type q-Szász-Mirakjan-Kantorovich operators and some approximation results. Carpathian J. Math. 2018, 34 (3), 363-370. doi:10.37193/CJM.2018.03.11

[22] Mursaleen M., Ansari K.J., Khan A. Approximation by Kantorovich type q-Bernstein Stancu operators. Complex Anal. Oper. Theory 2017, 11 (1), 85-107. doi:10.1007/s11785-016-0572-1

[23] Totik V. Uniform approximation by Szász-Mirakjan type operators. Acta Math. Hungar. 1983, 41 (3-4), $291-307$. doi:10.1007/bf01961317

[24] Totik V. Approximation by Szász-Mirakjan-Kantorovich operators in $L^{p}(p>1)$. Anal. Math. 1983, 9 (2), $147-167$. doi:10.1007/bf01982010

[25] Weierstrass K. Über die analytische Darstellbarkeit sogenannter willkürlicher Functionen einer reellen Veränderlichen Sitzungsberichteder. Königl. Preuss. Akad. Wiss. Berlin 1885, 2, 633-639.

Received 13.01.2021

Revised 13.03.2021

Касім М., Хан А., Аббас 3., Мурсалін М. Властивості збіжності узагальнених операторів Аупаша-Канторовича // Карпатські матем. публ. - 2021. - Т.13, №3. - С. 818-830.

У цій статті ми розглядаємо модифікацію Канторовича узагальнених операторів Аупаша, конструкція яких залежить від неперервно диференційовної зростаючої і необмеженої функції $\rho$. Аля цих нових операторів ми даємо зважену апроксимацію, теорему типу Вороновської, кількісні оцінки для локальної апроксимації.

Ключові слова і фрази: оператор Аупаша, оператор Канторовича, теорема типу Коровкіна, теорема про збіжність, теорема типу Вороновської. 